\title{
Comparison of Therapeutic Effect of Extracorporeal Shock Wave in Calcific Versus Noncalcific Lateral Epicondylopathy
}

\author{
Jong Wook Park, MD, Ji Hye Hwang, MD, PhD, Yoo Seong Choi, MD, Sang Jun Kim, MD, PhD
}

Department of Physical and Rehabilitation Medicine, Samsung Medical Center, Sungkyunkwan University School of Medicine, Seoul, Korea

Objective To assess the therapeutic effect of extracorporeal shock wave therapy (ESWT) in lateral epicondylopathy with calcification, and compare it to the effect of ESWT in lateral epicondylopathy without calcification.

Methods A retrospective study was conducted. Forty-three patients (19 with calcific and 24 with noncalcific lateral epicondylopathy in ultrasound imaging) were included. Clinical evaluations included the 100-point score, Nirschl Pain Phase scale before and after ESWT, and Roles and Maudsley (R\&M) scores after ESWT. ESWT (2,000 impulses and $0.06-0.12 \mathrm{~mJ} / \mathrm{mm}^{2}$ ) was performed once a week for 4 weeks.

Results The 100-point score and Nirschl Pain Phase scale changed significantly over time $(\mathrm{p}<0.001)$, but there was no significant difference between groups ( $p=0.555)$. The R\&M scores at 3 and 6 months after ESWT were not significantly different between groups. In the presence of a tendon tear, those in the calcific lateral epicondylopathy group showed poor improvement of 100-point scores compared to the noncalcific group $(\mathrm{p}=0.004)$.

Conclusion This study demonstrated that the therapeutic effect of ESWT in calcific lateral epicondylopathy was not significantly different from that in noncalcific lateral epicondylopathy. When a tendon tear is present, patients with calcific lateral epicondylopathy might show poor prognosis after ESWT relative to patients with noncalcific lateral epicondylopathy.

Keywords Epicondylitis, Extracorporeal shockwave

Received February 2, 2015; Accepted August 28, 2015

Corresponding author: Sang Jun Kim

Department of Physical and Rehabilitation Medicine, Samsung Medical Center, Sungkyunkwan University School of Medicine, 81 Irwon-ro, Gangnam-gu, Seoul 06351, Korea

Tel: +82-2-3410-6069, Fax: +82-2-3410-0052, E-mail: guitarren.kim@ samsung.com

(c) This is an open-access article distributed under the terms of the Creative Commons Attribution Non-Commercial License (http://creativecommons. org/licenses/by-nc/4.0) which permits unrestricted noncommercial use, distribution, and reproduction in any medium, provided the original work is properly cited.

Copyright $\odot 2016$ by Korean Academy of Rehabilitation Medicine

\section{INTRODUCTION}

Lateral epicondylopathy is a common disease with a reported incidence of $1 \%-3 \%$ in the general population, and its pathology is considered tendinosis rather than tendinitis [1]. The disease is thought to be self-limiting, but it can cause severe pain and can interfere with daily activities. Moreover, it has a tendency to recur if ignored and left untreated, and even after adequate treatment. Conservative treatments for lateral epicondylopathy include non-steroidal anti-inflammatory drugs, physical 
modalities, prolotherapy, steroid injection, and emerging treatments such as platelet-rich plasma injection [2] and stem cell injection [3]. Therapeutic success rates for lateral epicondylopathy vary depending on the treatment protocol and patients' elbow condition. Several investigators have tried to identify reasons for different success rates in the treatment of lateral epicondylopathy but thus far have been unsuccessful $[4,5]$.

Extracorporeal shock wave therapy (ESWT) is a treatment that uses focused or unfocused pulses that dissipate mechanical energy, and it is frequently used for the treatment of musculoskeletal diseases [6]. Though the exact therapeutic mechanism of shock wave therapy is unknown, it is associated with stimulation of the healing processes such as neovascularization, suppression of nociceptor activity and pain control mechanisms, and direct fragmentation of calcific deposits in the case of calcific tendinopathy [7]. A recent in vitro study [8] demonstrated that ESWT can promote growth of tenocytes and subsequent collagen synthesis, which suggests tendon healing may occur after the application of ESWT. However, not all patients with tendinopathy improve after the application of ESWT. In a recent systematic review [9], there was consistent evidence that ESWT was useful for the treatment of calcific tendinopathy of the rotator cuff and plantar fasciitis, but there was limited evidence that ESWT was useful for the treatment of Achilles tendinopathy and lateral epicondylopathy. Randomized controlled studies $[10,11]$ confirmed that ESWT did not show any superiority over the sham control group in treating chronic lateral epicondylopathy. The authors suggested that this inconsistent result might be due to diverse treatment regimens and tendon status.

Considering that calcific tendinitis of the shoulder responds well to ESWT [12] and histologic findings between rotator cuff tendinopathy and lateral epicondylopathy were similar in a previous study [13], we assumed that lateral epicondylopathy with calcification of the common extensor tendon will respond very well to ESWT relative to lateral epicondylopathy of the same tendon without calcification. However, there is no study assessing the therapeutic efficacy of ESWT in calcific lateral epicondylopathy. Therefore, we conducted this study to compare the therapeutic effect of ESWT on calcific lateral epicondylopathy and noncalcific lateral epicondylopathy.

\section{MATERIALS AND METHODS}

To evaluate the effect of ESWT, we conducted a retrospective study. This study included patients who were diagnosed with lateral epicondylopathy and visited our outpatient clinics to receive ESWT from January 2006 to December 2008. Patients were diagnosed with lateral epicondylopathy clinically (based on symptoms, site of tenderness, and pain elicited upon resisted active extension of the wrist in pronation and elbow extension). Selection criteria included 1) patients who had not received any injections including steroid injections within the past 3 months; 2) patients who had not taken any medication to relieve pain within the past 2 weeks; 3 ) patients who did not show any abnormal findings on simple radiologic examination except for calcification; 4) patients who did not have concomitant posterior neck pain. The patients who had concomitant posterior neck pain were excluded from this study because of the higher likelihood of cervical radiculopathy, which can aggravate the elbow pain; classically, these patients have not shown clinical improvement after ESWT. Among them, we included the cases in which ultrasound imaging studies and clinical evaluations were performed immediately prior to ESWT, 3 months after ESWT, and 6 months after ESWT, with not more than a 1-week variation. Clinical evaluations for measuring symptomatic improvement included the 100-point score, Nirschl Pain Phase Scale before and after ESWT, and Roles and Maudsley (R\&M) scores after ESWT. The 100-point scoring system includes pain (0-40), function (0-30), and strength (0-20) scores, as well as range of elbow motion (0-10), which was specified in a previous study [14]. The Nirschl Pain Phase scale and R\&M scores were evaluated as described in previous studies $[15,16]$. R\&M scores were rated as: 1 , excellent, no pain, full movement, full activity; 2, good, occasional discomfort, full movement, and full activity; 3 , fair, some discomfort after prolonged activity; and 4, poor, pain limits activities. A summary of the 100-point score, Nirschl Pain Phase scale, and R\&M scores is presented in a Supplementary Table 1.

Ultrasound evaluation was performed, and results were interpreted by two experienced physicians before ESWT. An ultrasound imaging system (Voluson E6; GE Healthcare, Milwaukee, WI, USA) with a linear array transducer was used to examine the extensor carpi radialis muscle 
Table 1. Baseline characteristics of patients with lateral epicondylopathy

\begin{tabular}{|c|c|c|}
\hline Group & $\begin{array}{c}\text { Calcific group } \\
(n=19)\end{array}$ & $\begin{array}{l}\text { acalcific group } \\
(\mathrm{n}=\mathbf{2 4})\end{array}$ \\
\hline Age (yr) & $50.7 \pm 2.1$ & $49.0 \pm 2.0$ \\
\hline Gender (male:female) & $2: 17$ & $9: 15$ \\
\hline $\begin{array}{l}\text { Laterality of } \\
\text { symptom (left:right) }\end{array}$ & $7: 12$ & 9:15 \\
\hline $\begin{array}{l}\text { Time from } \\
\text { symptom onset }\end{array}$ & $23.5 \pm 4.4$ & $29.0 \pm 6.2$ \\
\hline \multicolumn{3}{|l|}{ Ultrasound findings } \\
\hline $\begin{array}{l}\text { Diffuse } \\
\text { hypoechogenicity }\end{array}$ & $17 / 19(89.5)$ & $19 / 24(79.2)$ \\
\hline Tendon tear & 3/19 (15.8) & 6/24 (25.0) \\
\hline \multicolumn{3}{|l|}{ Values of clinical data } \\
\hline 100-point score & $46.4 \pm 4.0$ & $43.8 \pm 3.5$ \\
\hline Nirschl scale & $5.3 \pm 0.4$ & $5.0 \pm 0.4$ \\
\hline
\end{tabular}

Values are presented as average \pm standard error or number (\%).

and tendon. Ultrasound findings such as the presence of tendon tears, diffuse hypoechogenicity, and calcification were recorded. The presence of tendon tears was determined by focal areas of anechoic change with no intact fiber, or as distinct hypoechoic areas of fibril discontinuity. Calcification was determined by the presence of hyperechogenic matter with post-echogenic shadowing. Diffuse hypoechogenicity was defined as decreased echogenicity of the common extensor tendon compared to echogenicity of other tendons [17].

ESWT was performed once a week for 4 weeks using an electromagnetic shockwave generator (SONOCUR Basic; Siemens, Erlangen, Germany). Each session of the treatment consisted of 2,000 impulses of shockwave at 0.06$0.12 \mathrm{~mJ} / \mathrm{mm}^{2}$. The target area for application of ESWT was the most tender point on the lateral epicondyle, and it was adjusted during the treatment according to the patient's response. ESWT was used as first-line therapy for these patients, and the patients in this study did not receive any other medication for pain management or physical modalities except for self-stretching and isometric strengthening exercises of the extensor carpi radialis muscle during the ESWT sessions.

Patients were classified into calcific and noncalcific lateral epicondylopathy groups according to the results of the ultrasound. Temporal changes in the 100-point score after ESWT were considered to be the primary outcomes and temporal changes in the Nirschl Pain Phase scale after ESWT and R\&M scores at 3 and 6 months after ESWT were considered to be secondary outcomes. To compare the therapeutic effect of ESWT in calcific lateral epicondylopathy with that of ESWT in noncalcific lateral epicondylopathy, the repeated measures ANOVA test was adopted for the statistical analysis of the 100-point score and Nirschl Pain Phase scale, and the Wilcoxon signedrank sum test was adopted for the statistical analysis of R\&M scores. STATA ver. 12.0 (StataCorp LP, College Station, TX, USA) was used for the statistical analysis. All the patients provided informed consent to participate in this study. The study procedures were approved by our Institute Review Board (IRB No. 2010-12-082).

\section{RESULTS}

A total of 43 patients were included in this study. Nineteen patients were assigned to the calcific lateral epicondylopathy group and 24 patients were assigned to the noncalcific lateral epicondylopathy group. The average age was 50.7 years in the calcific lateral epicondylopathy group and 49.0 years in the noncalcific lateral epicondylopathy group, and this difference was not significant $(\mathrm{p}=0.558)$. The average time period between symptom onset and initial examination was 23.5 months in the calcific lateral epicondylopathy group and 29.0 months in the noncalcific lateral epicondylopathy group ( $\mathrm{p}=0.498$ ). The ratios of males to females and left to right involved sides were not significantly different between the two groups ( $p=0.077$ and $p=1.000)$. None of the patients suffered from bilateral epicondylopathy. There was no difference between tendon tear and diffuse hypoechogenicity between the two groups. The initial clinical data (100-point score and Nirschl Pain Phase scale) did not differ according to the presence of calcification. All of the baseline data are summarized in Table 1.

The temporal changes in the 100 -point score and Nirschl Pain Phase scale in the calcific and noncalcific lateral epicondylopathy groups are presented in Fig. 1. The 100-point score changed significantly over time $\left(\mathrm{F}_{(\mathrm{df}=1)}=97.801, \mathrm{p}<0.001\right)$, but there was no significant difference between groups $\left(\mathrm{F}_{(\mathrm{df}=1)}=0.355, \mathrm{p}=0.555\right)$. Nirschl Pain Phase scale also showed significant changes over time $\left(\mathrm{F}_{(\mathrm{df}=1)}=63.061, \mathrm{p}<0.001\right)$, but no significant 
(A)

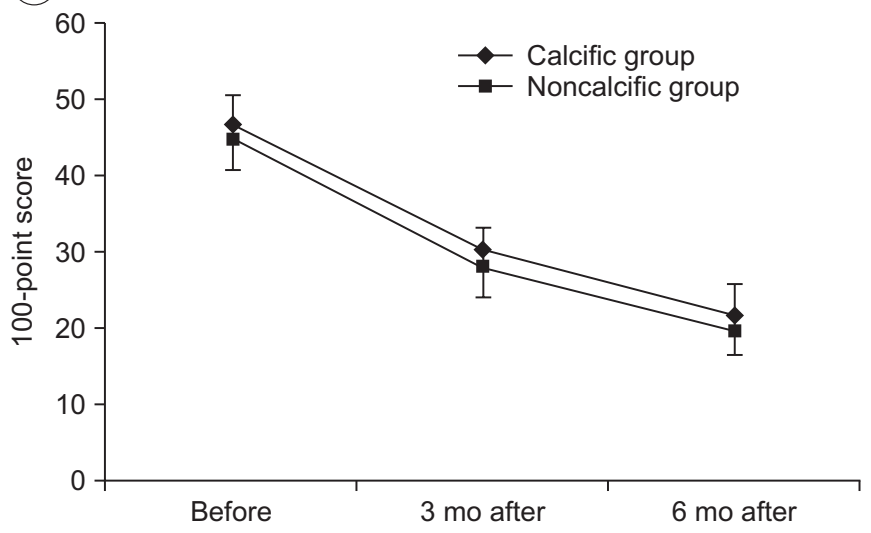

(B)

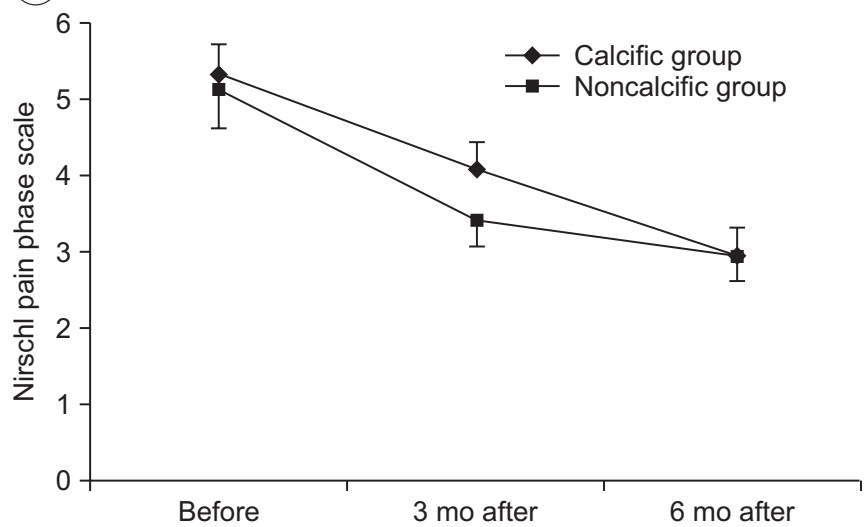

Fig. 1. The temporal changes in the 100-point scores (A) and Nirschl Pain Phase scale (B) in the calcific and noncalcific lateral epicondylopathy groups were presented. Error bars represent one standard error. The 100-point scores and Nirschl Pain Phase scale in the calcific and noncalcific groups changed significantly over time, but did not show any difference between groups.

differences were observed between groups $\left(\mathrm{F}_{(\mathrm{df}=1)}=0.311\right.$, $\mathrm{p}=0.581) . R \& \mathrm{M}$ scores in the calcific and noncalcific lateral epicondylopathy groups were 2.84 and 2.52 , respectively, at 3 months after ESWT, and these scores decreased to 2.32 and 2.21, respectively, at 6 months after ESWT (Fig. 2). The R\&M scores at 3 and 6 months after ESWT did not differ significantly between groups $(\mathrm{p}=0.250$ and $\mathrm{p}=0.653$, Wilcoxon signed-rank sum test).

When the patients were analyzed according to the other ultrasound findings (diffuse hypoechogenicity and tendon tear), only those patients of the calcific and noncalcific lateral epicondylopathy groups who had a tendon tear showed a difference in temporal changes in the 100-point score $\left(\mathrm{F}_{(\mathrm{df}=1)}=20.577, \mathrm{p}=0.004\right)$. Also, the calcific group showed poor improvement of 100-point scores compared to the noncalcific group (Fig. 3).

The amount of change in the 100-point score was calculated by subtracting the 100-point scores at 3 and 6 months after ESWT from the 100-point scores before ESWT.

\section{DISCUSSION}

In this study, we found that patients with calcific lateral epicondylopathy did not show a significantly different response to ESWT than patients with noncalcific lateral epicondylopathy. When a tendon tear is present, patients with calcific lateral epicondylopathy might show a poor prognosis after ESWT than patients with noncalcific lat-

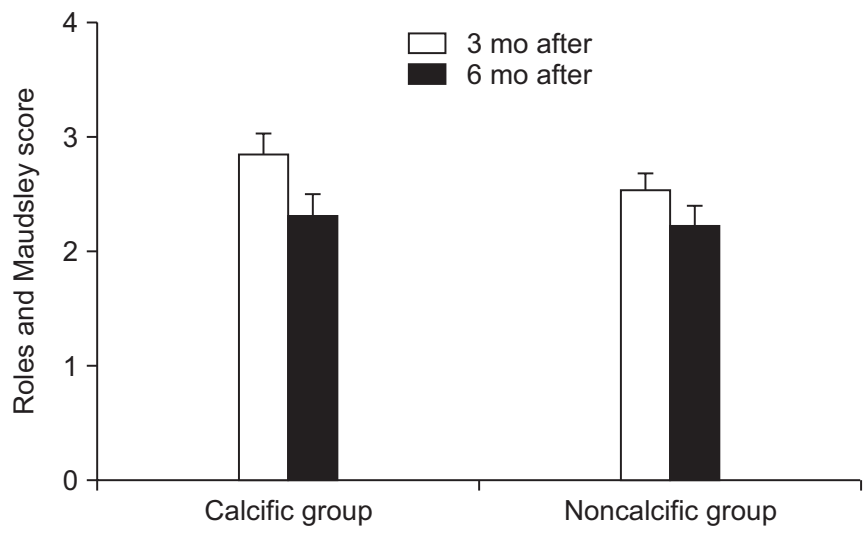

Fig. 2. Roles and Maudsley scores in the calcific and noncalcific groups at 3 and 6 months after extracorporeal shock wave therapy were presented. The Roles and Maudsley scores at 3 and 6 months were not significantly different between groups.

eral epicondylopathy. The findings obtained from this study can assist clinicians who treat patients with lateral epicondylopathy in the decision to use ESWT for treating this condition.

This study treated the clinical data obtained at 3 and 6 months after ESWT as the primary outcome, because this time period was thought to be adequate for assessing tendon healing in chronic tendinopathy [18]. In our study, patients with chronic lateral epicondylopathy showed clinical improvement after ESWT, irrespective of the presence of calcification. This might be due to the fact that the therapeutic mechanism of ESWT in tendi- 
(A)

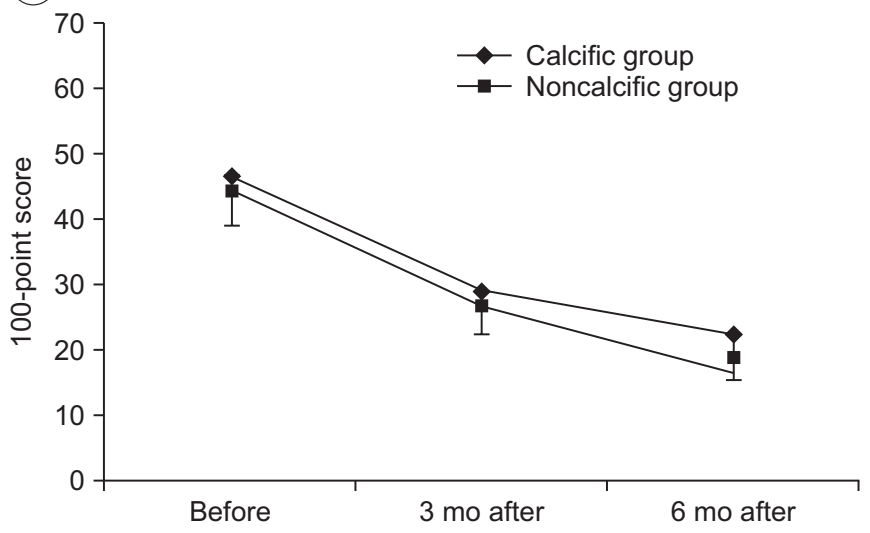

(C)

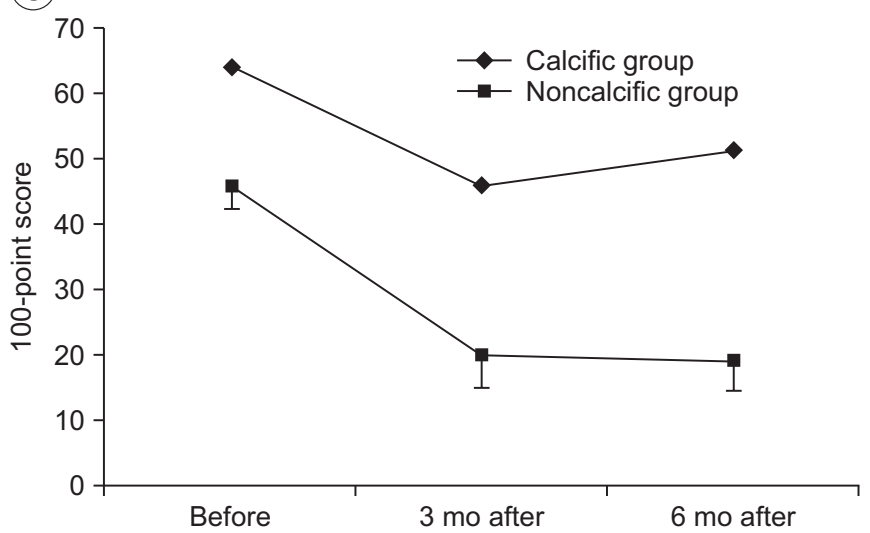

(B)

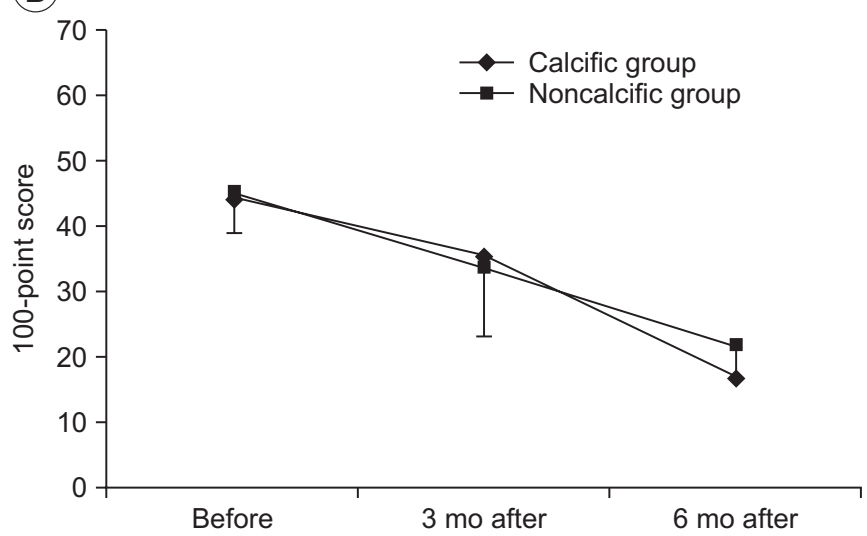

(D)

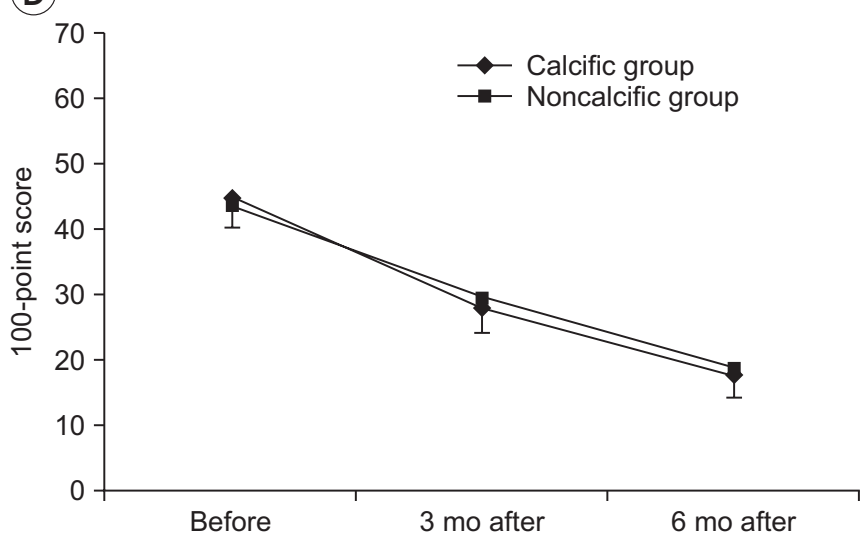

Fig. 3. The temporal changes in the 100-point scores were analyzed according to the ultrasound findings: (A) hypoechogenicity, (B) no hypoechogenicity, (C) tendon tear, and (D) no tendon tear. Patients of the calcific and noncalcific lateral epicondylopathy groups who had a tendon tear showed a difference in temporal changes in the 100-point score and the calcific group showed poor improvement of 100-point scores compared to the noncalcific group.

nopathy includes not only the degradation of calcific deposits but also a decrease in elevated inflammatory cytokines that are observed in tendinopathy [19], denervation of pain receptors, and increased tissue healing by neovascularization [7], which are not related to calcification. However, we must also consider the possibility of spontaneous recovery in patients with chronic lateral epicondylopathy or the placebo effect of ESWT because sham treatment was not employed in this study.

In contrast with our initial hypothesis, ESWT did not show a better outcome in calcific lateral epicondylopathy than in noncalcific lateral epicondylopathy. This result was not consistent with the results of previous studies, which stated that the resorption of calcifications was important in determining whether ESWT would be effective $[20,21]$. Considering that the response of calcific rotator cuff tendinitis to ESWT is different from the response of calcific lateral epicondylopathy to ESWT, there might be a difference in the role of calcification between calcific rotator cuff tendinitis and calcific lateral epicondylopathy. In addition, calcifications might not be a major cause of pain and discomfort in patients with lateral epicondylopathy, although we did not assess the resorption of calcifications by simple radiology or follow-up ultrasound images. Other possible cause of the different result might come from stimulus site. In this study, we treated ESWT at the site where maximal local pain was reproduced according to the protocols of previous studies. The purpose of the study was to compare responses according to the existence of calcification findings on ultrasound. We followed the previous method of ESWT on lateral epicondylopathy. However, recently, in treatment of calcific tendinitis of the shoulder, ESWT focused on the calcified region rather than insertion site of the tendon has been 
reported to be more effective [22]. Therefore, if we conducted therapy specifically on calcific site instead of at the tender site of the tendon, the therapeutic effect might be different. Further study is required to analyze the effectiveness of ESWT according to the focused calcific site.

In this study, tendon tear was shown to be linked with poor outcome in patients with calcific lateral epicondylopathy. We speculated that calcification due to chondral metaplasia after a tendon tear [23] would be different from calcification without tendon tear, and it might hinder the clinical improvement after ESWT.

In a previous study of histological and ultrastructural findings in calcific lateral epicondylopathy, tenocytes in calcific lesions seemed to be well preserved and maintained collagen function and turnover ability [24]. The results suggested that tendon tissue repair could occur in calcific lesions of epicondylopathy. We cannot clearly explain the reason that calcific lateral epicondylopathy with tendon tear showed resistance to treatment. However, considering the results of the previous study, repetitive focal trauma causing linear tendon tear might inhibit the tenocyte action of tissue repair and cause the resistance to the ESWT in calcific lateral epicondylopathy. There are no studies on the comparison of characteristics of calcification associated in the presence or absence of tendon tear or tendon tear effects to calcific lesions. To clarify this assumption, further in vitro studies are needed.

Our study has several limitations. First, this study was conducted as a retrospective study. To obtain more accurate results, prospective further study should be done. Second, the therapeutic effect of ESWT in the tendon tear group was postulated from a small number of patients. Third, two patients showed both diffuse hypoechogenicity and tendon tear features upon ultrasound imaging. Therefore, in future studies, to identify the effect of ESWT relative to ultrasound findings, we should exclude patients who have multiple characteristic ultrasound findings.

In conclusion, this study demonstrated that the therapeutic effect of ESWT in calcific lateral epicondylopathy was not superior to that of noncalcific lateral epicondylopathy. When a tendon tear is present, patients with calcific lateral epicondylopathy might have a poorer prognosis after ESWT than patients with noncalcific lateral epicondylopathy. The findings obtained from this study will be useful for making a decision on whether to use
ESWT for treating lateral epicondylopathy. Based on the results of the study, if a patient has tendon tear combined with calcification, other conservative therapies should be considered in place of ESWT. However, additional studies utilizing larger sample sizes and further assessment of the pathophysiology associated with epicondylopathy are necessary.

\section{CONFLICT OF INTEREST}

No potential conflict of interest relevant to this article was reported.

\section{SUPPLEMENTARY MATERIALS}

Supplementary materials can be found via http:// dx.doi.org/10.5535/arm.2016.40.2.294. Table S1. Summary of 100-point scores, Nirschl Phase Rating scale, and Roles and Maudsley scores.

\section{REFERENCES}

1. Tosti R, Jennings J, Sewards JM. Lateral epicondylitis of the elbow. Am J Med 2013;126:357.e1-6.

2. Mishra A, Collado H, Fredericson M. Platelet-rich plasma compared with corticosteroid injection for chronic lateral elbow tendinosis. PM R 2009;1:366-70.

3. Connell D, Datir A, Alyas F, Curtis M. Treatment of lateral epicondylitis using skin-derived tenocyte-like cells. Br J Sports Med 2009;43:293-8.

4. Struijs PA, Spruyt M, Assendelft WJ, van Dijk CN. The predictive value of diagnostic sonography for the effectiveness of conservative treatment of tennis elbow. AJR Am J Roentgenol 2005;185:1113-8.

5. Vicenzino B, Smith D, Cleland J, Bisset L. Development of a clinical prediction rule to identify initial responders to mobilisation with movement and exercise for lateral epicondylalgia. Man Ther 2009;14:550-4.

6. Foldager CB, Kearney C, Spector M. Clinical application of extracorporeal shock wave therapy in orthopedics: focused versus unfocused shock waves. Ultrasound Med Biol 2012;38:1673-80.

7. Mouzopoulos G, Stamatakos M, Mouzopoulos D, Tzurbakis M. Extracorporeal shock wave treatment for shoulder calcific tendonitis: a systematic review. Skeletal Radiol 2007;36:803-11. 
8. Vetrano M, d'Alessandro F, Torrisi MR, Ferretti A, Vulpiani MC, Visco V. Extracorporeal shock wave therapy promotes cell proliferation and collagen synthesis of primary cultured human tenocytes. Knee Surg Sports Traumatol Arthrosc 2011;19:2159-68.

9. Speed C. A systematic review of shockwave therapies in soft tissue conditions: focusing on the evidence. $\mathrm{Br}$ J Sports Med 2014;48:1538-42.

10. Melikyan EY, Shahin E, Miles J, Bainbridge LC. Extracorporeal shock-wave treatment for tennis elbow: a randomised double-blind study. J Bone Joint Surg Br 2003;85:852-5.

11. Speed CA, Nichols D, Richards C, Humphreys H, Wies JT, Burnet S, et al. Extracorporeal shock wave therapy for lateral epicondylitis: a double blind randomised controlled trial. J Orthop Res 2002;20:895-8.

12. Peters J, Luboldt W, Schwarz W, Jacobi V, Herzog C, Vogl TJ. Extracorporeal shock wave therapy in calcific tendinitis of the shoulder. Skeletal Radiol 2004;33:7128.

13. Chard MD, Cawston TE, Riley GP, Gresham GA, Hazleman BL. Rotator cuffdegeneration and lateral epicondylitis: a comparative histological study. Ann Rheum Dis 1994;53:30-4.

14. Wang CJ, Chen HS. Shock wave therapy for patients with lateral epicondylitis of the elbow: a one- to twoyear follow-up study. Am J Sports Med 2002;30:422-5.

15. Cho BK, Kim YM, Kim DS, Choi ES, Shon HC, Park KJ, et al. Mini-open muscle resection procedure under local anesthesia for lateral and medial epicondylitis. Clin Orthop Surg 2009;1:123-7.

16. Nirschl RP. Elbow tendinosis/tennis elbow. Clin
Sports Med 1992;11:851-70.

17. Clarke AW, Ahmad M, Curtis M, Connell DA. Lateral elbow tendinopathy: correlation of ultrasound findings with pain and functional disability. Am J Sports Med 2010;38:1209-14.

18. Al-Abbad H, Simon JV. The effectiveness of extracorporeal shock wave therapy on chronic Achilles tendinopathy: a systematic review. Foot Ankle Int 2013; 34:33-41.

19. Han SH, Lee JW, Guyton GP, Parks BG, Courneya JP, Schon LC. J.Leonard Goldner Award 2008. Effect of extracorporeal shock wave therapy on cultured tenocytes. Foot Ankle Int 2009;30:93-8.

20. Carcia CR, Scibek JS. Causation and management of calcific tendonitis and periarthritis. Curr Opin Rheumatol 2013;25:204-9.

21. Harniman E, Carette S, Kennedy C, Beaton D. Extracorporeal shock wave therapy for calcific and noncalcific tendonitis of the rotator cuff: a systematic review. J Hand Ther 2004;17:132-51.

22. Haake M, Deike B, Thon A, Schmitt J. Value of exact focusing of extracorporeal shock waves (ESWT) in therapy of tendinitis calcarea: a prospective randomized study. Biomed Tech (Berl) 2001;46:69-74.

23. Hashimoto T, Nobuhara K, Hamada T. Pathologic evidence of degeneration as a primary cause of rotator cuff tear. Clin Orthop Relat Res 2003;415:111-20.

24. Galliani I, Columbaro M, Ferri S, Valmori A, Cassiani G, Falcieri E. A case of calcific lateral epicondylitis: a histological and ultrastructural study. Br J Rheumatol 1998;37:235-6. 